\title{
DESENVOLVIMENTO FOLIAR EM DUAS CULTIVARES DE OLIVEIRA ESTIMADO POR DUAS CATEGORIAS DE MODELOS
}

\author{
FABRINA BOLZAN MARTINS ${ }^{1}$, RODOLFO ARMANDO DE ALMEIDA PEREIRA ${ }^{1}$, MARCOS \\ VINÍCIUS MARQUES PINHEIRO ${ }^{2}$, MARCEL CARVALHO ABREU ${ }^{1}$
}

\author{
${ }^{1}$ Universidade Federal de Itajubá, Instituto de Recursos Naturais, Itajubá, MG, Brasil \\ ${ }^{2}$ Universidade Federal de Viçosa, Instituto de Biotecnologia aplicada à Agropecuária, Viçosa, MG, Brasil \\ fabrinabm@gmail.com,rodolfoarmando.17@gmail.com,macvini@gmail.com,marcelc.abreu@gmail.com
}

Recebido Fevereiro de 2014 - Aceito Junho de 2014

\begin{abstract}
RESUMO
Os modelos de simulação são ferramentas utilizadas na área de agrometeorologia para quantificar o desenvolvimento de culturas agrícolas e florestais. O trabalho teve como objetivo comparar os modelos do Filocrono (linear) e de Wang e Engel (não linear) para estimar o desenvolvimento foliar, através do aparecimento de folhas, nas cultivares de oliveira 'Arbequina' e 'MGS ASC315' durante a fase de muda. $\mathrm{O}$ experimento foi instalado no campo experimental da Empresa Agropecuária de Minas Gerais (EPAMIG), Maria da Fé (MG), sob o delineamento inteiramente casualizado e organizado em arranjo fatorial, sendo duas cultivares e nove épocas de transplantio com 15 repetições em cada tratamento. Os coeficientes dos modelos foram estimados pelos dados obtidos nas três primeiras épocas de transplantio e as demais épocas foram utilizadas para avaliar o desempenho dos modelos. As respostas dos modelos foram divergentes para os dois modelos. O modelo de Wang e Engel foi mais eficiente para a cultivar 'Arbequina', com raiz do quadrado médio do erro (RQME) de 3,41 folhas, enquanto a cultivar 'MGS ASC315' teve a RQME de 6,58 folhas. O modelo do Filocrono se mostrou mais eficiente para a cultivar 'MG ASC315' com RQME de 2,98 folhas, inferior ao da cultivar 'Arbequina', com RQME de 7,49 folhas.

Palavras-chave: Olea europaea, aparecimento de folhas, desenvolvimento vegetal, Filocrono, Wang e Engel.

ABSTRACT: LEAF DEVELOPMENT IN TWO OLIVE CULTIVARS ESTIMATED BY TWO MODELS.

Simulation models are tools used in the Agrometeorology to quantify the development of agricultural and forest crops. The aim of this work was to evaluate and compare the Phyllochron (linear) and the Wang and Engel (non-linear) models for estimating the leaf development, by leaf appearance, in two olive cultivars during the seedling phase. A field experiment was carried out in the Agricultural Company of Minas Gerais (EPAMIG), Maria da Fé (MG). The experimental design was completely randomized in a factorial arrangement, with two cultivars, nine planting dates and 15 repetitions in each treatment. The three first planting dates were used to estimate the models coefficients and other planting dates were used as independent data to evaluate the models. The response was different for the two cultivars. The Wang and Engel model estimations performed better for 'Arbequina', with a Root Mean Square Error (RMSE) of 3.41 leaves while the Phyllochron model better estimated for 'MGS ASC315'with RMSE of 2.98 leaves, below the one obtained for the 'Arbequina' cultivar with a RMSE of 7.49 leaves.
\end{abstract}

Keywords: Olea europaea L., leaf appearance, plant development, Phyllochron, Wang and Engel. 


\section{INTRODUÇÃO}

A espécie Olea europaea L., conhecida popularmente como oliveira, é uma árvore frutífera subtropical de grande importância devido à utilização do azeite e da azeitona na dieta humana (Kitsaki et al., 2010), sendo estes produtos ricos em antioxidantes, além de possuir propriedades que diminuem os riscos de doenças cardiovasculares (Goulas et al., 2009).

A oliveira é cultivada em diversas regiões do mundo, porém sua produção está concentrada em alguns países da Europa (EMBRAPA, 2009), principalmente na região do mediterrâneo (Denney et al., 1985). Na América do Sul, a Argentina e o Chile são destaques na produção e exportação de azeite (EMBRAPA, 2009), enquanto no Brasil o cultivo de oliveira ainda é incipiente, com pequenos plantios principalmente nas regiões Sul e Sudeste, devido as condições climáticas serem distintas do seu centro de origem (Martins et al., 2012). Por esse motivo, o Brasil importa quantidade superior a 64 mil toneladas de produtos oriundos da oliveira (IOC, 2012), sendo o terceiro maior importador mundial de azeite e o segundo maior importador mundial de azeitonas de mesa (Teramoto et al., 2013).

Para viabilizar o cultivo dessa cultura no Brasil, faz-se necessário conhecer os fatores bióticos e abióticos que influenciam o desenvolvimento vegetativo e reprodutivo da espécie. Dentre os fatores abióticos a temperatura do ar é considerado o fator mais importante para o desenvolvimento e produtividade da oliveira (Denney et al., 1985; Ayerza e Sibbett, 2001; Martins et al., 2012), o que justifica a necessidade de estudos que associem a temperatura com parâmetros de desenvolvimento. A taxa de aparecimento de folhas (TAF), número de folhas acumuladas na haste principal (NF), dias do calendário civil e duração dos estágios de desenvolvimento são parâmetros de desenvolvimento vegetal bastante utilizados nesse tipo de estudo (Streck et al., 2009).

Uma forma tradicional de relacionar a temperatura do ar com parâmetros de desenvolvimento é através dos modelos de simulação amplamente usados em culturas anuais (Rosa et al., 2009, Streck et al., 2009) e perenes (Martins e Streck, 2007), os quais são ferramentas comuns na área de Agrometeorologia. Estes modelos integram a velocidade de emissão de folhas, representado pela taxa de aparecimento de folhas (TAF), em relação ao tempo, sendo tempo, neste caso, dado por funções de temperatura (Martins et al., 2007). Existem duas categorias de modelos de simulação: linear ou aditiva e não linear ou multiplicativa. Na categoria linear o modelo do Filocrono é o exemplo mais recorrente, enquanto o modelo de Wang e Engel (WE) é bastante utilizado na categoria não linear (Wang e Engel, 1998; Xue et al., 2004; Evers, et al., 2007; Streck et al., 2008a; Streck et al., 2009; Lisboa et al., 2012).
O modelo do Filocrono propõe uma relação linear e aditiva entre a TAF e a função de temperatura do ar, a qual é expressa por meio da soma térmica, cuja unidade é ${ }^{\circ} \mathrm{C}$ dia (Streck et al., 2005; Xue et al., 2004; Evers, et al., 2007; Martins et al., 2007; Rosa et al., 2009). O modelo do Filocrono é preferido por ser o mais simples de implementar, tem menor número de coeficientes, sendo funcional para muitas situações práticas (Xue et al., 2004; Streck et al., 2009). Porém, o modelo do Filocrono é alvo de algumas críticas, entre elas a pressuposição de que a relação entre o desenvolvimento da planta é linearmente proporcional à temperatura (Yan e Hunt, 1999; Xue et al., 2004; Martins et al., 2007), o que contraria muitos processos biológicos, incluindo o desenvolvimento de plantas através da TAF, de ter tendência não linear (Streck et al, 2005).

Outra vertente propõe uma relação não linear entre o desenvolvimento vegetal e a temperatura do ar. Entre eles, o modelo de WE tem sido bastante utilizado para simular o desenvolvimento de muitas culturas (Streck et al., 2008a), pois permite associar de forma multiplicativa funções de resposta à temperatura $[\mathrm{f}(\mathrm{t})]$ com a TAF (Wang e Engel, 1998; Xue et al., 2004; Martins e Streck, 2007; Streck et al., 2008b). No modelo de WE, a função de resposta à temperatura do ar é dada por uma função beta (Wang e Engel, 1998).

Modelos não lineares ou multiplicativos como o de WE são considerados mais realísticos do ponto de vista biológico do que modelos lineares ou aditivos, como o Filocrono. Porém, não há confirmação da superioridade de um modelo sobre o outro. Estudos têm sido realizados em diversas culturas anuais (Evers et al., 2007; Streck et al., 2008a; Streck et al., 2008b; Streck et al., 2009 e perenes (Martins e Streck, 2007) com diferentes categorias de modelos para simular o desenvolvimento e outros estudos objetivam comparar e selecionar a melhor categoria de modelo para simular o desenvolvimento de diferentes culturas. Porém, informações relativas ao desempenho de modelos de simulação de desenvolvimento vegetal em oliveira são ainda inexistentes, revelando a importância do estudo proposto para a espécie, genótipos e condições climáticas locais contempladas neste estudo.

O objetivo deste trabalho foi avaliar e comparar os modelos do Filocrono e de Wang e Engel para estimativa do desenvolvimento foliar nas cultivares de $O$. europaea $\mathrm{L}$. 'Arbequina' e 'MGS ASC315' durante a fase de muda.

\section{MATERIAL E MÉTODOS}

Para estimar o desenvolvimento foliar, representado pelas variáveis NF e TAF, foram utilizados os modelos Filocrono (Xue et al., 2004; Streck et al., 2008a) e WE (Wang e Engel, 1998). A representação dos dois modelos pode ser visualizada na Figura 1. 

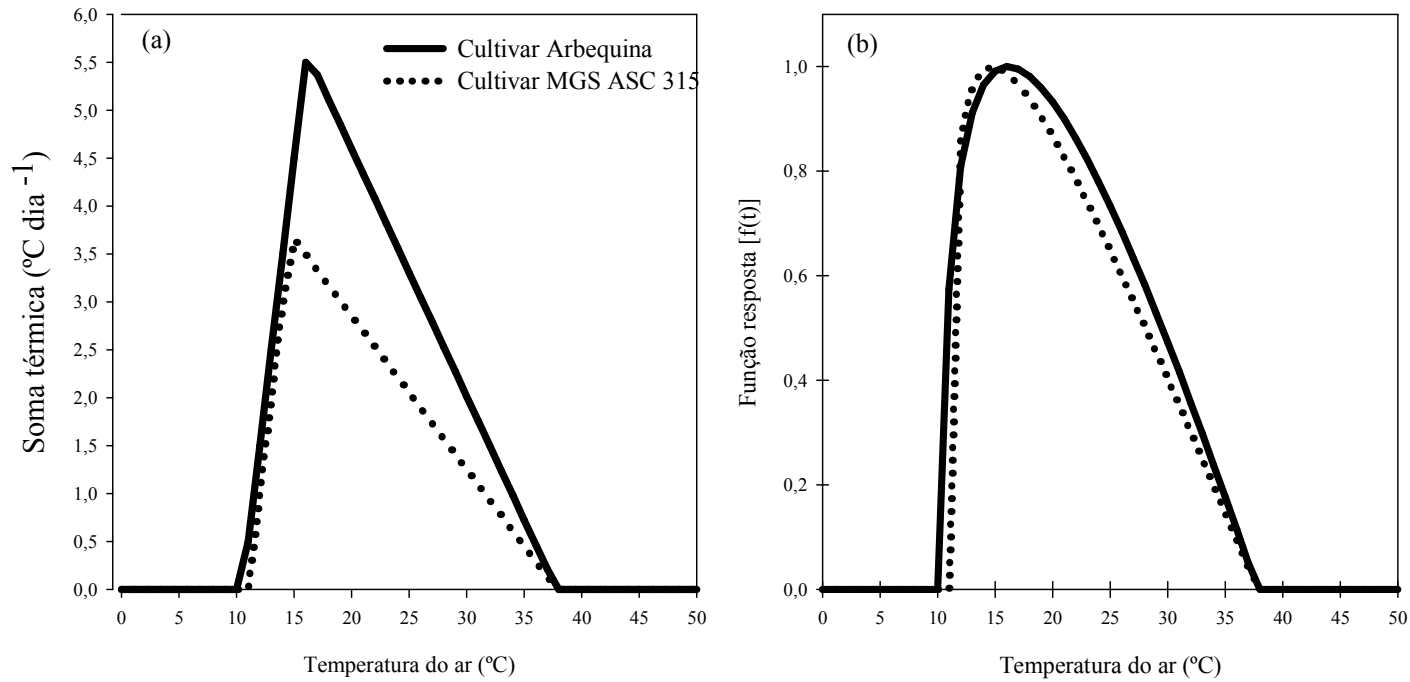

Figura 1 - Representação gráfica do método de cálculo da soma térmica usado no modelo do Filocrono (a) e curva da função resposta usada no modelo de Wang e Engel (b) para as duas cultivares de oliveira.

O modelo do Filocrono é determinado pelo inverso do coeficiente angular (a) da regressão linear entre NF e soma térmica acumulada $\left(\mathrm{STa},{ }^{\circ} \mathrm{C}\right.$ dia) a partir da data de transplantio (Xue et al., 2004): $N F=a \cdot S T a+b$; sendo que a STa é calculada pelo somatório da soma térmica diária (STd), dada por (Streck, 2002; Martins e Streck, 2007): $S T d=T m-T b .1$ dia: Quando $\mathrm{Tb}<\mathrm{Tm} \leq \mathrm{Tot}$; e $S T d=(T o t-T b)$. [(Tm - Tmax $) /(T o t-$ $T b$ )].1dia: Quando Tot $<\mathrm{Tm} \leq \mathrm{Tmax} . \mathrm{Se}: \mathrm{Tm} \leq \mathrm{Tb}, \mathrm{STd}=0$; se: $\mathrm{Tm} \geq \mathrm{Tmax} ; \mathrm{STd}=0$, em que: $\mathrm{Tm}=$ temperatura média diária do ar, dada pela média aritmética das temperaturas máxima e mínima diárias do $\operatorname{ar}\left({ }^{\circ} \mathrm{C}\right) ; \mathrm{Tb}$, Tot e Tmax são as temperaturas cardinais de desenvolvimento para cada cultivar (mínima, ótima e máxima, respectivamente). Para a 'Arbequina', as temperaturas cardinais são: $\mathrm{Tb}=10,5^{\circ} \mathrm{C}$ (Martins et al., 2012), Tot $=16,1^{\circ} \mathrm{C}$ (Lisboa et al., 2012). Para a 'MGS ASC315' são: $\mathrm{Tb}=11^{\circ} \mathrm{C}$ (Martins et al., 2012), $\mathrm{Tot}=14,7^{\circ} \mathrm{C}$ (Lisboa et al., 2012). Como não foi encontrado um valor especifico de Tmax para cada cultivar, utilizou-se o valor de $37,8^{\circ} \mathrm{C}$ (Denney et al., 1985), para ambas cultivares.

Pelo modelo do Filocrono, calcula-se NF por: $\mathrm{NF}=\mathrm{STa} /$ Filocrono (Martins e Streck, 2007; Streck et al., 2008b).

O modelo de WE (Wang e Engel, 1998) utiliza uma função de resposta não linear de temperatura $\mathrm{f}(\mathrm{t})$, conhecida por função beta, combinada com um fator multiplicativo (TAFmax) dada por: TAF $=$ TAF $\max f(t)$, em que a TAF é a taxa de aparecimento de folhas (folhas por dia).

A f $(\mathrm{t})$ é uma função beta definida como:

$$
f(T)=\frac{\left[2 \cdot(T m-T b)^{\alpha}(T o t-T b)^{\alpha}-(T m-T b)^{2 \alpha}\right]}{(T o t-T b)^{2 \alpha}}
$$

quando $\mathrm{Tb}<\mathrm{Tm} \leq \mathrm{Tmax}$. Se $\mathrm{Tm}<\mathrm{Tb}$ e se $\mathrm{Tm}>\mathrm{Tmax} ; \mathrm{f}(\mathrm{t})=0$.

$$
\alpha=\frac{\ln 2}{\ln [(T \max -T b) /(T o t-T b)]}
$$

em que $\mathrm{Tb}$, Tot e Tmax = temperaturas cardinais, mínima, ótima e máxima de cada cultivar $\left({ }^{\circ} \mathrm{C}\right)$, respectivamente; $\mathrm{Tm}=$ temperatura média diária do ar $\left({ }^{\circ} \mathrm{C}\right)$. Os valores de $\mathrm{Tb}$, Tot e Tmax são os mesmos do modelo do Filocrono.

No WE, para o cálculo do NF, somam-se os valores de TAF diários, a partir da data de transplantio.

Para obter dados do desenvolvimento foliar das duas cultivares de oliveira foi instalado um experimento a campo na área da fazenda experimental da Empresa Agropecuária de Minas Gerais (EPAMIG), localizada no município de Maria da Fé (MG), latitude $22^{\circ} 18^{\prime} 29^{\prime \prime} \mathrm{S}$, longitude $45^{\circ} 22^{\prime} 31^{\prime \prime}$ e altitude de 1.276 m. A classificação do clima do local, segundo Köeppen, é $\mathrm{Cwb}$, subtropical de altitude, caracterizado por invernos secos e verões chuvosos de temperaturas inferiores a $22^{\circ} \mathrm{C}$ (Vieira Neto et al., 2011; Martins et al., 2012).

$\mathrm{O}$ experimento foi instalado em delineamento inteiramente casualizado, em esquema fatorial $2 \times 9$, sendo duas cultivares de oliveira ('Arbequina' e 'MGS ASC315') e nove épocas de transplantio, composto por 15 repetições em cada tratamento. A unidade experimental foi caracterizada por estaca semilenhosa de oliveira enraizada em vaso de polietileno de aproximadamente $4 \mathrm{~L}$, preenchidos com substrato constituído de terra e composto orgânico comercial (Provaso ${ }^{\circledR}$ ). Cada $\mathrm{m}^{3}$ de substrato foi enriquecido com $5 \mathrm{~kg}$ de superfosfato simples (18 dag kg ${ }^{-1}$ de $\left.\mathrm{P}_{2} \mathrm{O}_{5}\right), 1 \mathrm{~kg}$ de cloreto de potássio (58 dag $\mathrm{kg}^{-1}$ de $\mathrm{K}_{2} \mathrm{O}$ ) e 2,5 kg de calcário dolomítico (PRNT 100\%).

Para minimizar a influência da radiação solar, e assim evitar um aumento de temperatura do substrato, os vasos foram 
envolvidos com papel jornal a fim de reduzir o que poderia ser uma fonte de erro experimental (Martins et al., 2007). O espaço entre os vasos foi de aproximadamente $20 \mathrm{~cm}$ de forma a não sombrear os vasos vizinhos, e realizou-se regas regulares para que as plantas não sofressem estresse hídrico.

Utilizaram-se mudas provenientes do enraizamento de estacas semilenhosas e ausentes de brotações das cultivares de oliveira Arbequina e MGS ASC315. O método de estaquia foi utilizado devido ao lento processo de germinação das sementes de oliveira (Acebedo et al., 1997).

As nove épocas de transplantio foram instaladas em intervalos de aproximadamente 30 dias, expondo as plantas a diferentes condições meteorológicas durante o seu desenvolvimento (Rosa et al., 2009). As diferentes datas de instalação foram: época 1 (E1) - 20/05/2010, época 2 (E2) - 20/06/2010, época 3 (E3) - 20/07/2010, época 4 (E4) - 20/08/2010, época 5 (E5) - 29/09/2010, época 6 (E6) - 27/10/2010, época 7 (E7) - 20/11/2010, época 8 (E8) 21/12/2010 e época 9 (E9) - 15/01/2011. As épocas 1,2 e 3 foram utilizadas para estimar os coeficientes do modelo de Filocrono e WE. As demais épocas foram utilizadas para simular e avaliar o desempenho dos dois modelos.

O coeficiente 'a' foi estimado através da média aritmética dos coeficientes angulares das regressões lineares para cada cultivar (15 regressões para cada época e cultivar) entre NF e STa. O coeficiente "TAFmax" foi obtido com procedimento nonlinear estimation do software Statistica (Statsoft, 2008), que utiliza uma variante do método Gauss-Newton pelo método dos mínimos quadrados para estimar os parâmetros da regressão não linear entre valores do NF acumulado em função dos valores acumulados de $\mathrm{f}(\mathrm{t})$. $\mathrm{O}$ valor do 'TAFmax'é aquele da regressão que forneceu o menor valor do quadrado médio do erro (QME) (Martins e Streck, 2007).

Foram obtidos dados diários de temperatura mínima e máxima do ar através de uma estação meteorológica convencional pertencente ao Instituto Mineiro de Gestão das Águas (IGAM), localizada na EPAMIG, distante $100 \mathrm{~m}$ do local de instalação do experimento.

O NF emitido na haste principal foi contado semanalmente, a partir do momento em que havia uma folha visível na haste principal. Foi considerada folha visível somente quando o comprimento alcançou no mínimo de $1 \mathrm{~cm}$ (Lisboa et al., 2012). Para a sua contagem, a haste principal recebeu um anel colorido. $\mathrm{O}$ término da fase de muda foi considerado quando cada repetição tinha 20 folhas visíveis (Lisboa et al., 2012).

Para avaliar o desempenho dos modelos foram testadas normalidade e homogeneidade da variância entre o NF observado e estimado pelos dois modelos, através do teste de Bartlett; comparação de médias entre o NF observado e estimado, pelo teste t. Os desvios da estimativa do NF foi avaliado pelo teste $\mathrm{F}$, no qual a relação entre o quadrado médio do erro (QME) dos dois modelos, dado por

$$
\sum_{i=1}^{n}(E i-O i)^{2} / n
$$

$(\mathrm{Ei}=\mathrm{NF}$ estimados e $\mathrm{Oi}=\mathrm{NF}$ observados $)$, segue a distribuição de F, ou seja,

\section{$\left.\left(\mathrm{QME}_{\mathrm{Fil}} / \mathrm{QME}_{\mathrm{WE}}\right) \cap \mathrm{F}\right)$}

Outras estatísticas utilizadas para avaliar o desempenho dos modelos foram: raiz do quadrado médio do erro (RQME) (Streck et al., 2008a), BIAS (Martins e Streck, 2007), coeficiente de correlação (r), índice de concordância 'd', índice 'c' (Camargo e Sentelhas, 1997).

O RQME expressa o erro médio do modelo, e quanto menor, melhor é o modelo (Streck et al., 2008ab):

$$
R Q M E=\left[\sum_{i=1}^{n}(E i-O i)^{2} / n\right]^{0,5}
$$

em que Ei são os valores de NF estimados pelos modelos; Oi são os valores de NF observados; n é o número de observações.

O índice BIAS expressa a tendência do modelo, onde leva em consideração o desvio médio dos valores estimados em relação aos valores observados. Quanto menor o valor de BIAS menos tendencioso e melhor é o modelo (Leite e Andrade, 2002):

$$
B I A S=\sum_{i=1}^{n} E i-\sum_{i=1}^{n} O i / \sum_{i=1}^{n} O i
$$

A estatística $r$ determina o grau de associação entre duas ou mais variáveis, sendo que quanto mais próximo de 1 (positivo ou negativo), maior a relação entre valores observados e estimados e melhor é o modelo (Martins e Streck, 2007):

$$
r=\sum_{i=1}^{n}(O i-\bar{O})(E i-\bar{E}) /\left\{\left[\sum_{i=1}^{n}(O i-\bar{O})^{2}\right]\left[\sum_{i=1}^{n}(E i-\bar{E})^{2}\right]\right\}^{0,5}
$$

em que: $\overline{\mathrm{E}}=$ média dos valores estimados; $\overline{\mathrm{O}}=$ média dos valores observados.

$\mathrm{O}$ 'd' representa o quanto os valores estimados estão livres de erro, e variam de 0 a 1 , sendo que quanto mais próximo de 1, maior a concordância entre os valores estimados e observados (Willmott, 1981):

$$
d=1-\left\{\left[\sum_{i=1}^{n}(E i-O i)^{2}\right] /\left[\sum_{i=1}^{n}(|E i-\bar{O}|+|O i-\bar{O}|)^{2}\right]\right\}
$$

O índice ' $c$ ' fornece o desempenho do modelo e sua classificação é dada na Tabela 1, em conformidade com o proposto por Camargo e Sentelhas (1997), sendo obtido pelo produto entre $\mathrm{r}$ e d.

A comparação entre os dois modelos foi feita através do valor ponderado (Vp) dos escores estatísticos (Martins e Streck, 
2007). Para tal, foi atribuído para cada indicador estatístico um peso (1 ou 2), no qual o melhor indicador estatístico recebeu peso 1 , enquanto o pior recebeu peso 2 . O somatório dos pesos atribuídos a cada indicador estatístico constituiu o Vp e, quanto menor o seu valor, melhor é o modelo.

\section{RESULTADOS E DISCUSSÃO}

As temperaturas médias da máxima e mínima do ar oscilaram durante as nove épocas de experimento (Tabela 2). Em termos absolutos, o menor valor de temperatura do ar foi de $-1,3^{\circ} \mathrm{C}$, registrada durante as épocas E2, E3 e E4, enquanto o maior valor absoluto de temperatura do ar foi de $31,4^{\circ} \mathrm{C}$ durante as épocas E5, E6 e E7. As diferenças de condições de temperatura do ar afetaram a velocidade de emissão de folhas na haste principal, o que é considerado um importante fator quando se estuda o crescimento e o desenvolvimento vegetal (Rosa et al., 2009), além de ser importante na estimativa dos coeficientes e na avaliação dos dois modelos estatísticos para a estimativa da TAF (Streck et al., 2003; Streck et al., 2008a).

Dentre as épocas do experimento, a cultivar 'Arbequina' teve maior velocidade de emissão de folhas durante E4, uma vez que a duração da época ocorreu em menor número de dias (64 dias) (Tabela 2), devido ao maior número de dias com temperaturas próximas da temperatura ótima para a cultivar, e menor velocidade de emissão durante a E2 (100 dias), quando as temperaturas foram menores. Já a cultivar 'MGS ASC315' teve maior velocidade de emissão de folhas durante a E1 (84 dias) e menor durante a E2 (170 dias), quando as temperaturas do ar estavam mais amenas. Mesmo assim, a 'MGS ASC315' emitiu folhas mais lentamente que a 'Arbequina' em todas as épocas (Tabela 3).

$\mathrm{O}$ valor do coeficiente "a" do modelo de Filocrono foi de 0,06580 (Filocrono $=15,20^{\circ} \mathrm{C}$ dia por folha) para a cultivar 'Arbequina' e 0,05866 para a cultivar 'MGS ASC $315^{\prime}$ ' (Filocrono $=17,05^{\circ} \mathrm{C}$ dia por folha). $\mathrm{O}$ coeficiente "TAFmax" do modelo de WE foi de 0,270390 folhas $\mathrm{dia}^{-1}$ para a 'Arbequina' e 0,272980 folhas $\mathrm{dia}^{-1}$ para 'MGS ASC315',

Tabela 1 - Critérios para interpretação do desempenho dos modelos pelo índice c (Camargo e Sentelhas, 1997).

\begin{tabular}{ll}
\hline Valor de c & Desempenho \\
\hline$>0,85$ & Ótimo \\
0,76 a 0,85 & Muito bom \\
0,66 a 0,75 & Bom \\
0,61 a 0,65 & Moderado \\
0,51 a 0,60 & Fraco \\
0,41 a 0,50 & Muito fraco \\
$\leq 0,40$ & Péssimo \\
\hline
\end{tabular}

sendo ambos coeficientes estimados através das três primeiras épocas de transplantio (E1, E2 e E3).

Pelo teste $t$, aplicado para comparar a média entre o NF observado com as médias estimadas pelos dois modelos, observa-se que para 'Arbequina', exceto para E5, os valores de NF observados foram semelhantes ao NF estimados pelos dois modelos (Tabela 3). Para a 'Arbequina' a média do NF estimado foi sempre maior com o modelo do Filocrono comparado ao modelo de WE. Já para a cultivar 'MGS ASC315', o modelo do Filocrono não apresentou diferença significativa em nenhuma época, enquanto o modelo de WE, apresentou diferença significativa nas E4, E5, E6 e E8. Para a 'MGS ASC315' a média do NF estimado foi superior a média do NF observada com o modelo de WE comparado ao modelo do Filocrono. Para a 'Arbequina' a média do NF estimado pelo modelo do Filocrono foi superior a média do NF observado nas seis épocas, e somente em uma época para o modelo de WE, enquanto que para a 'MGS ASC315' tanto o Filocrono quanto o WE foram superiores ao NF observado nas seis épocas.

$\mathrm{O}$ teste de Bartlett indicou que os pressupostos da normalidade e homogeneidade foram atendidos para os valores de NF observados e estimados pelos dois modelos e para as duas cultivares, o que é desejável do ponto de vista biológico e estatístico, pois os dados são homocedásticos (possuem variância constante) (Martins e Streck, 2007).

Os desvios da estimativa do NF pelos dois modelos foram testados assumindo-se que o QME segue a distribuição de F. Nesse sentido, o QME foi menor para o modelo do Filocrono em todas as épocas para a 'MGS ASC315', enquanto na 'Arbequina' não foi detectada diferença estatística entre os modelos. Estes resultados indicam um melhor resultado para o modelo do Filocrono para a cultivar 'MGS ASC315' em relação ao modelo WE, e que não há diferença estatística entre os desvios dos dois modelos para a cultivar 'Arbequina'(Tabela $3)$.

As estatísticas analisadas para as duas cultivares (Tabela 4), confirmam que para a 'Arbequina', apesar dos valores estatísticos serem relativamente próximos, houve ligeira melhora do desempenho do modelo do Filocrono para a E4 $(\mathrm{Vp}=5)$, e do modelo de WE para as demais épocas, enquanto que para a 'MGS ASC315' o modelo do Filocrono foi melhor para todas as épocas em que foi feita a validação.

Para a 'MGS ASC315' o valor do RQME foi menor para o modelo do Filocrono nas seis épocas de transplantio, enquanto que a 'Arbequina' o modelo de WE, exceto para a E4, apresentou menor valor do RQME. A 'MSG ASC315' também apresentou as estatísticas BIAS; $\mathrm{r}$; $\mathrm{d}$; e c melhores para o modelo do Filocrono em relação ao modelo de WE em todas as épocas. Para a 'Arbequina' as estatísticas BIAS; r; d; e c, no geral, também foram ligeiramente melhores para o modelo de WE. 
Tabela 2 - Média das temperaturas mínimas, médias e máximas do ar e duração das nove épocas de transplantio, a qual corresponde ao período desde a data de instalação de cada época até a última observação do NF. Maria da Fé (MG) 2010/2011.

\begin{tabular}{cccccc}
\hline \multirow{2}{*}{ Época } & \multicolumn{2}{c}{$\begin{array}{c}\text { Média da temperatura do ar } \\
\left({ }^{\circ} \mathrm{C}\right)\end{array}$} & \multicolumn{2}{c}{$\begin{array}{c}\text { Duração da fase de } \\
\text { desenvolvimento (dias) }\end{array}$} \\
\cline { 2 - 6 } & Mínima & Média & Máxima & Arbequina & $\begin{array}{c}\text { MGS } \\
\text { ASC315 }\end{array}$ \\
\hline E1 & 6,3 & 14,4 & 22,5 & 69 & 84 \\
\hline E2 & 5,8 & 14,6 & 23,4 & 100 & 170 \\
\hline E3 & 7,4 & 16,1 & 24,8 & 76 & 132 \\
\hline E4 & 10,4 & 18,0 & 25,5 & 64 & 126 \\
\hline E5 & 13,0 & 19,6 & 25,8 & 91 & 110 \\
\hline E6 & 13,6 & 20,6 & 26,6 & 69 & 110 \\
\hline E7 & 15,5 & 21,3 & 27,1 & 72 & 100 \\
\hline E8 & 15,6 & 21,5 & 26,8 & 70 & 98 \\
\hline E9 & 14,2 & 20,4 & 26,5 & 89 & 108 \\
\hline & & & & &
\end{tabular}

Tabela 3 - Média, variância e quadrado médio do erro referente ao número observado de folhas acumuladas na haste principal (OBS), usadas na avaliação do desempenho dos modelos do Filocrono (FIL) e de Wang e Engel (WE) para a estimativa do aparecimento de folhas em duas cultivares de oliveira. Maria da Fé (MG) 2010/2011.

\begin{tabular}{|c|c|c|c|c|c|c|c|}
\hline \multirow{2}{*}{ Estatísticas } & \multirow{2}{*}{$\begin{array}{l}\text { Número de } \\
\text { folhas }\end{array}$} & \multicolumn{6}{|c|}{ 'Arbequina' } \\
\hline & & E4 & E5 & E6 & E7 & E8 & E9 \\
\hline \multirow{3}{*}{ Média } & OBS & 8,23 & 9,28 & 9,53 & 10,50 & 9,97 & 8,89 \\
\hline & FIL & $8,75^{\text {ns }}$ & $15,31^{*}$ & $12,51^{\mathrm{ns}}$ & $11,74^{\mathrm{ns}}$ & $11,10^{\mathrm{ns}}$ & $10,38^{\mathrm{ns}}$ \\
\hline & WE & $7,86^{\mathrm{ns}}$ & $12,64^{\mathrm{ns}}$ & $9,51^{\mathrm{ns}}$ & $9,94^{\mathrm{ns}}$ & $9,24^{\mathrm{ns}}$ & $8,35^{\text {ns }}$ \\
\hline \multirow{3}{*}{$\begin{array}{l}\text { Desvio } \\
\text { Padrão }\end{array}$} & OBS & 8,94 & 8,11 & 8,44 & 9,22 & 9,08 & 8,08 \\
\hline & FIL & $6,15^{\text {ns }}$ & $8,30^{\mathrm{ns}}$ & $6,89^{\mathrm{ns}}$ & $9,95^{\text {ns }}$ & $9,58^{\mathrm{ns}}$ & $8,04^{\mathrm{ns}}$ \\
\hline & WE & $5,25^{\mathrm{ns}}$ & $6,83^{\mathrm{ns}}$ & $5,74^{\text {ns }}$ & $8,96^{\text {ns }}$ & $9,01^{\mathrm{ns}}$ & $7,53^{\text {ns }}$ \\
\hline \multirow{3}{*}{ QME } & FIL & 10,07 & 87,39 & 9,17 & 24,44 & 20,31 & 23,57 \\
\hline & WE & 15,06 & 47,61 & 8,85 & 7,05 & 11,04 & 7,87 \\
\hline & $\mathrm{F}$ & ns & ns & ns & ns & ns & $\mathrm{ns}$ \\
\hline & & \multicolumn{6}{|c|}{ 'MGS ASC 315' } \\
\hline \multirow{3}{*}{ Média } & OBS & 7,73 & 8,46 & 6,51 & 9,16 & 7,06 & 9,03 \\
\hline & FIL & $10,86^{\text {ns }}$ & $9,48^{\mathrm{ns}}$ & $10,06^{\mathrm{ns}}$ & $10,89^{\text {ns }}$ & $9,34^{\mathrm{ns}}$ & $9,48^{\mathrm{ns}}$ \\
\hline & WE & $15,42^{*}$ & $14,66^{*}$ & $13,91^{*}$ & $15,02^{\text {ns }}$ & $12,19^{*}$ & $14,66^{\mathrm{ns}}$ \\
\hline \multirow{3}{*}{$\begin{array}{l}\text { Desvio } \\
\text { Padrão }\end{array}$} & OBS & 6,47 & 6,02 & 6,22 & 7,33 & 6,43 & 6,13 \\
\hline & FIL & $6,64^{\mathrm{ns}}$ & $5,86^{\mathrm{ns}}$ & $5,51^{\mathrm{ns}}$ & $8,47^{\text {ns }}$ & $7,14^{\mathrm{ns}}$ & $5,86^{\mathrm{ns}}$ \\
\hline & WE & $9,32^{\mathrm{ns}}$ & $7,91^{\mathrm{ns}}$ & $7,81^{\mathrm{ns}}$ & $10,04^{\text {ns }}$ & $7,60^{\mathrm{ns}}$ & $8,99^{\text {ns }}$ \\
\hline \multirow{3}{*}{ QME } & FIL & 11,27 & 2,06 & 17,37 & 4,56 & 5,93 & 1,15 \\
\hline & WE & 68,95 & 43,72 & 63,01 & 42,18 & 29,36 & 57,78 \\
\hline & $\mathrm{F}$ & ns & ns & ns & ns & ns & \\
\hline
\end{tabular}

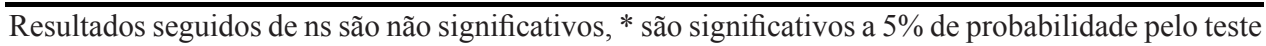
t, que compara a média observada com cada média estimada; pelo teste de Barlett, que compara a variância observada com a estimada; e pelo teste F, que compara o QME dos dois modelos testados. 
Tabela 4 - Estatísticas utilizadas para avaliação dos modelos do Filocrono (FIL) e de Wang e Engel (WE) para a estimativa do aparecimento de folhas nas cultivares 'Arbequina' e 'MGS ASC315', em seis épocas de transplantio (E4, E5, E6, E7, E8 e E9), Maria da Fé, MG 2010/2011.

\begin{tabular}{|c|c|c|c|c|c|c|c|c|c|c|c|c|}
\hline \multirow{3}{*}{ Estatística } & \multicolumn{12}{|c|}{ Época/modelo } \\
\hline & \multicolumn{2}{|c|}{ E4 } & \multicolumn{2}{|c|}{ E5 } & \multicolumn{2}{|c|}{ E6 } & \multicolumn{2}{|c|}{ E7 } & \multicolumn{2}{|c|}{ E8 } & \multicolumn{2}{|c|}{ E9 } \\
\hline & FIL & WE & FIL & WE & FIL & WE & FIL & WE & FIL & WE & FIL & WE \\
\hline & \multicolumn{12}{|c|}{ 'Arbequina' } \\
\hline RQME & $\begin{array}{c}3,1730 \\
\text { (1) }\end{array}$ & $\begin{array}{c}3,8806 \\
(2)\end{array}$ & $\begin{array}{c}9,3483 \\
(2)\end{array}$ & $\begin{array}{c}6,8999 \\
(1)\end{array}$ & $\begin{array}{c}3,0276 \\
(2)\end{array}$ & $\begin{array}{c}2,9760 \\
\text { (1) }\end{array}$ & $\begin{array}{c}2,5036 \\
\text { (2) }\end{array}$ & $\begin{array}{c}1,8015 \\
\text { (1) }\end{array}$ & $\begin{array}{c}5,4315 \\
(2)\end{array}$ & $\begin{array}{c}4,0062 \\
\text { (1) }\end{array}$ & $\begin{array}{c}2,6453 \\
(2)\end{array}$ & $\begin{array}{c}0,9492 \\
\text { (1) }\end{array}$ \\
\hline BIAS & $\begin{array}{c}0,0631 \\
(1)\end{array}$ & $\begin{array}{c}-0,0458 \\
\text { (2) }\end{array}$ & $\begin{array}{c}1,252 \\
(2)\end{array}$ & $\begin{array}{c}0,859 \\
(1)\end{array}$ & $\begin{array}{c}0,2085 \\
(2)\end{array}$ & $\begin{array}{c}-0,0017 \\
\text { (1) }\end{array}$ & $\begin{array}{c}0,1229 \\
(2)\end{array}$ & $\begin{array}{c}-0,0525 \\
\text { (1) }\end{array}$ & $\begin{array}{c}0,1355 \\
(2)\end{array}$ & $\begin{array}{c}-0,0648 \\
\text { (1) }\end{array}$ & $\begin{array}{c}0,1797 \\
(2)\end{array}$ & $\begin{array}{c}-0,0538 \\
\text { (1) }\end{array}$ \\
\hline $\mathrm{r}$ & $\begin{array}{c}0,9637 \\
(1)\end{array}$ & $\begin{array}{c}0,9540 \\
\text { (2) }\end{array}$ & $\begin{array}{c}0,8728 \\
\text { (2) }\end{array}$ & $\begin{array}{c}0,8745 \\
\text { (1) }\end{array}$ & $\begin{array}{c}0,9663 \\
\text { (2) }\end{array}$ & $\begin{array}{c}0,9671 \\
\text { (1) }\end{array}$ & $\begin{array}{c}0,9887 \\
\text { (2) }\end{array}$ & $\begin{array}{c}0,9981 \\
\text { (1) }\end{array}$ & $\begin{array}{c}0,9969 \\
\text { (1) }\end{array}$ & $\begin{array}{c}0,9961 \\
\text { (2) }\end{array}$ & $\begin{array}{c}0,9992 \\
\text { (1) }\end{array}$ & $\begin{array}{c}0,9968 \\
\text { (2) }\end{array}$ \\
\hline d & $\begin{array}{c}0,9044 \\
(1)\end{array}$ & $\begin{array}{c}0,8680 \\
\text { (2) }\end{array}$ & $\begin{array}{c}0,6970 \\
\text { (2) }\end{array}$ & $\begin{array}{c}0,7553 \\
\text { (1) }\end{array}$ & $\begin{array}{c}0,9020 \\
\text { (1) }\end{array}$ & $\begin{array}{c}0,8959 \\
(2)\end{array}$ & $\begin{array}{c}0,8969 \\
(2)\end{array}$ & $\begin{array}{c}0,90212 \\
\text { (1) }\end{array}$ & $\begin{array}{c}0,9215 \\
\text { (2) }\end{array}$ & $\begin{array}{c}0,9965 \\
\text { (1) }\end{array}$ & $\begin{array}{c}0,9021 \\
(2)\end{array}$ & $\begin{array}{c}0,9958 \\
\text { (1) }\end{array}$ \\
\hline $\mathrm{c}$ & $\begin{array}{c}0,8715 \\
\text { (1) }\end{array}$ & $\begin{array}{c}0,8281 \\
(2)\end{array}$ & $\begin{array}{c}0,6083 \\
(2)\end{array}$ & $\begin{array}{c}0,6606 \\
(1)\end{array}$ & $\begin{array}{c}0,8716 \\
\text { (1) }\end{array}$ & $\begin{array}{c}0,8664 \\
(2)\end{array}$ & $\begin{array}{c}0,8868 \\
(2)\end{array}$ & $\begin{array}{c}0,9004 \\
\text { (1) }\end{array}$ & $\begin{array}{c}0,9186 \\
(2)\end{array}$ & $\begin{array}{c}0,9926 \\
\text { (1) }\end{array}$ & $\begin{array}{c}0,9014 \\
(2)\end{array}$ & $\begin{array}{c}0,9927 \\
\text { (1) }\end{array}$ \\
\hline Vp & 5 & 10 & 10 & 5 & 8 & 7 & 10 & 5 & 9 & 6 & 9 & 6 \\
\hline & \multicolumn{12}{|c|}{ 'MGS ASC315' } \\
\hline RQME & $\begin{array}{c}3,3565 \\
\text { (1) }\end{array}$ & $\begin{array}{c}8,3035 \\
(2)\end{array}$ & $\begin{array}{c}1,4355 \\
\text { (1) }\end{array}$ & $\begin{array}{c}6,612 \\
(2)\end{array}$ & $\begin{array}{c}4,1673 \\
(1)\end{array}$ & $\begin{array}{c}7,9382 \\
\text { (2) }\end{array}$ & $\begin{array}{c}2,1108 \\
\text { (1) }\end{array}$ & $\begin{array}{c}6,4950 \\
(2)\end{array}$ & $\begin{array}{c}2,4344 \\
\text { (1) }\end{array}$ & $\begin{array}{c}5,4188 \\
\text { (2) }\end{array}$ & $\begin{array}{c}1,0711 \\
(1)\end{array}$ & $\begin{array}{c}7,6013 \\
(2)\end{array}$ \\
\hline BIAS & $\begin{array}{c}0,4039 \\
\text { (1) }\end{array}$ & $\begin{array}{c}0,9942 \\
\text { (2) }\end{array}$ & $\begin{array}{c}0,1208 \\
\text { (1) }\end{array}$ & $\begin{array}{c}0,7332 \\
\text { (2) }\end{array}$ & $\begin{array}{c}0,5455 \\
\text { (1) }\end{array}$ & $\begin{array}{c}1,1376 \\
\text { (2) }\end{array}$ & $\begin{array}{c}0,1903 \\
\text { (1) }\end{array}$ & $\begin{array}{c}0,6516 \\
(2)\end{array}$ & $\begin{array}{c}0,3241 \\
\text { (1) }\end{array}$ & $\begin{array}{c}0,7273 \\
\text { (2) }\end{array}$ & $\begin{array}{c}0,4969 \\
\text { (1) }\end{array}$ & $\begin{array}{c}0,7446 \\
\text { (2) }\end{array}$ \\
\hline $\mathrm{r}$ & $\begin{array}{c}0,9791 \\
\text { (1) }\end{array}$ & $\begin{array}{c}0,9773 \\
\text { (2) }\end{array}$ & $\begin{array}{c}0,9833 \\
\text { (1) }\end{array}$ & $\begin{array}{c}0,9738 \\
(2)\end{array}$ & $\begin{array}{c}0,9284 \\
(2)\end{array}$ & $\begin{array}{c}0,931 \\
(1)\end{array}$ & $\begin{array}{c}0,9976 \\
\text { (1) }\end{array}$ & $\begin{array}{c}0,9941 \\
(2)\end{array}$ & $\begin{array}{c}0,9974 \\
(1)\end{array}$ & $\begin{array}{c}0,9809 \\
(2)\end{array}$ & $\begin{array}{c}0,9868 \\
(1)\end{array}$ & $\begin{array}{c}0,9852 \\
(2)\end{array}$ \\
\hline d & $\begin{array}{c}0,8697 \\
(1)\end{array}$ & $\begin{array}{c}0,7146 \\
\text { (2) }\end{array}$ & $\begin{array}{c}0,9122 \\
\text { (1) }\end{array}$ & $\begin{array}{c}0,7373 \\
(2)\end{array}$ & $\begin{array}{c}0,8076 \\
(1)\end{array}$ & $\begin{array}{c}0,6917 \\
(2)\end{array}$ & $\begin{array}{c}0,9804 \\
(1)\end{array}$ & $\begin{array}{c}0,8625 \\
\text { (2) }\end{array}$ & $\begin{array}{c}0,9666 \\
(1)\end{array}$ & $\begin{array}{c}0,8633 \\
(2)\end{array}$ & $\begin{array}{c}0,9913 \\
\text { (1) }\end{array}$ & $\begin{array}{c}0,7917 \\
(2)\end{array}$ \\
\hline $\mathrm{c}$ & $\begin{array}{c}0,8516 \\
\text { (1) }\end{array}$ & $\begin{array}{c}0,6984 \\
\text { (2) }\end{array}$ & $\begin{array}{c}0,897 \\
(1)\end{array}$ & $\begin{array}{c}0,718 \\
(2)\end{array}$ & $\begin{array}{c}0,7498 \\
\text { (1) }\end{array}$ & $\begin{array}{c}0,644 \\
(2)\end{array}$ & $\begin{array}{c}0,9780 \\
\text { (1) }\end{array}$ & $\begin{array}{c}0,8575 \\
(2)\end{array}$ & $\begin{array}{c}0,9641 \\
(1)\end{array}$ & $\begin{array}{c}0,8469 \\
(2)\end{array}$ & $\begin{array}{c}0,9783 \\
\text { (1) }\end{array}$ & $\begin{array}{c}0,7800 \\
(2)\end{array}$ \\
\hline$\overline{V p}$ & 5 & 10 & 5 & 10 & 6 & 9 & 5 & 10 & 5 & 10 & 5 & 10 \\
\hline
\end{tabular}

Os valores entre parênteses, abaixo das estatísticas, são atribuídos para o valor do índice Vp. O valor 1 é atribuído ao melhor modelo em cada indicador estatístico, enquanto o valor 2 é atribuído ao pior modelo.

Os dados das seis épocas usadas na validação foram agrupados para comparar o NF observado e estimado pelos dois modelos (Figura 2). Pela análise visual, observa-se que o modelo de WE estimou melhor o NF do que o modelo do Filocrono para a 'Arbequina'. O valor de RQME global para o WE foi de 3,41 folhas (Figura 2b), com pequena superestimativa no NF, principalmente na $\mathrm{E} 5$ e na região com $\mathrm{NF}<10$, e de 7,49 folhas para o Filocrono, com a maioria dos dados de NF sendo superestimados (Figura 2a). Ou seja, o modelo de WE erra globalmente menos que o modelo do Filocrono, representando uma diferença média de $54,47 \%$. Na E5 houve um aumento considerável da temperatura do ar em relação as demais épocas. Porém, no início da E5 houve variação entre dias com temperatura do ar amenas e inferiores a $10,5^{\circ} \mathrm{C}(\mathrm{Tb}$ da 'Arbequina') e dias com temperatura do ar próxima a Tot da 'Arbequina', o que pode ter influenciado no desempenho modesto dos modelos na E5 com aumento do valor da TAF e consequentemente na superestimativa do NF. Além disso, por motivos alheios a nossa vontade, as medições no NF da E5 não ocorreram em intervalos regulares de tempo como nas demais épocas.

Já para 'MGS ASC315' o modelo do Filocrono estimou melhor o NF que o de WE, com RQME global de 2,98 folhas e 6,58 folhas, respectivamente. Observa-se que, apesar do modelo do Filocrono apresentar pequena superestimativa do NF, ele se aproxima mais da linha 1:1 (Figura 2c) e erra globalmente menos que o modelo de WE (diferença em torno de 54,71\%).

É importante salientar que modelos de desenvolvimento apresentam um erro quando há um pequeno número de folhas (Martins e Streck, 2007; Streck et al., 2010), já que não há tendência de comportamento bem definida no início do desenvolvimento vegetal (Soltani e Sinclair, 2012). Mesmo com essa dificuldade em captar o comportamento no início do desenvolvimento a RQME global foi inferior a 3,5 para as duas cultivares. Um erro de três a cinco folhas pode ser considerado baixo e aceitável (Martins e Streck, 2007; Streck et al., 2007b), inclusive em mudas de oliveira, as quais costumam ter suas folhas emitidas aos pares em intervalos entre 7 a 10 dias. Isso significa um erro de dois ou três nós e, em dias do calendário civil, de 10 a 15 dias. Para entender o que esses valores de erro representam no sentido prático é necessário verificar a quantidade de energia, em ${ }^{\circ} \mathrm{C}$ dia, que esse erro representa. Por exemplo, a duração da fase de muda é calculada baseada no Filocrono e assumindo NF de 20 (padrão utilizado para a fase de muda neste estudo) um erro de 3 folhas representa um erro de $45,6^{\circ} \mathrm{C}$ dia para a 'Arbequina', enquanto um erro de 3 folhas representa um erro de $51^{\circ} \mathrm{C}$ dia para a 'MGS ASC315', o qual representa poucos dias do calendário civil. Além disso, 

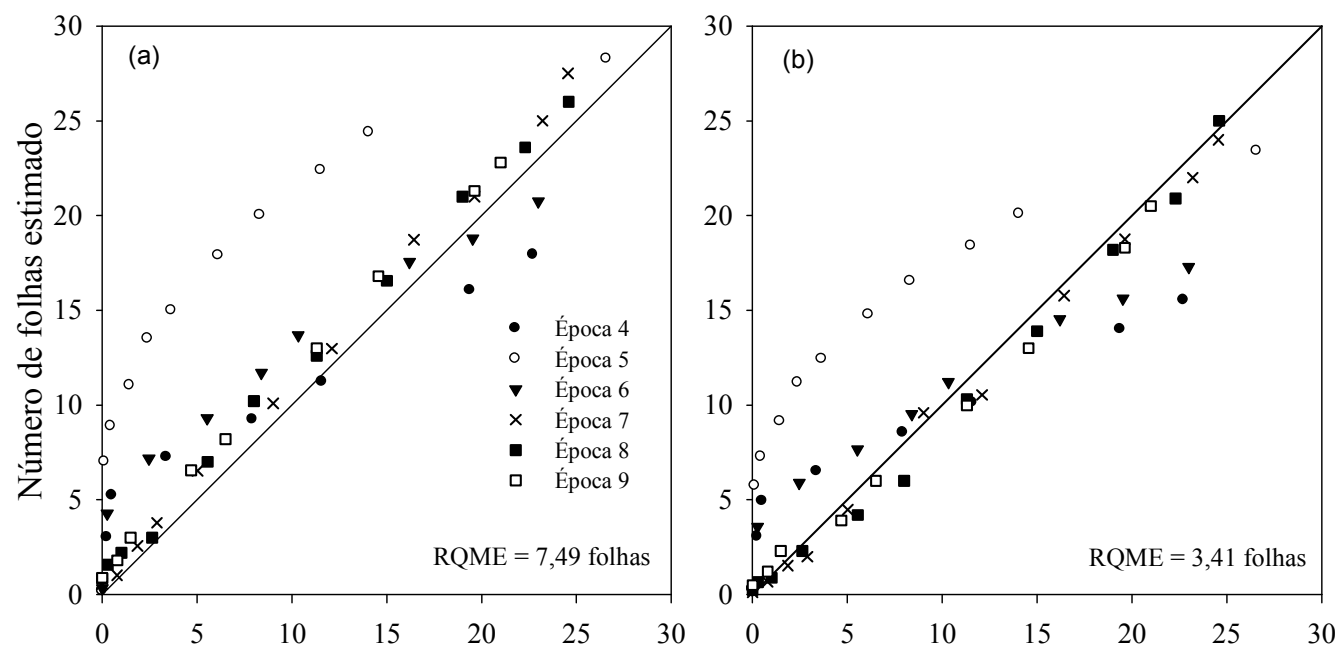

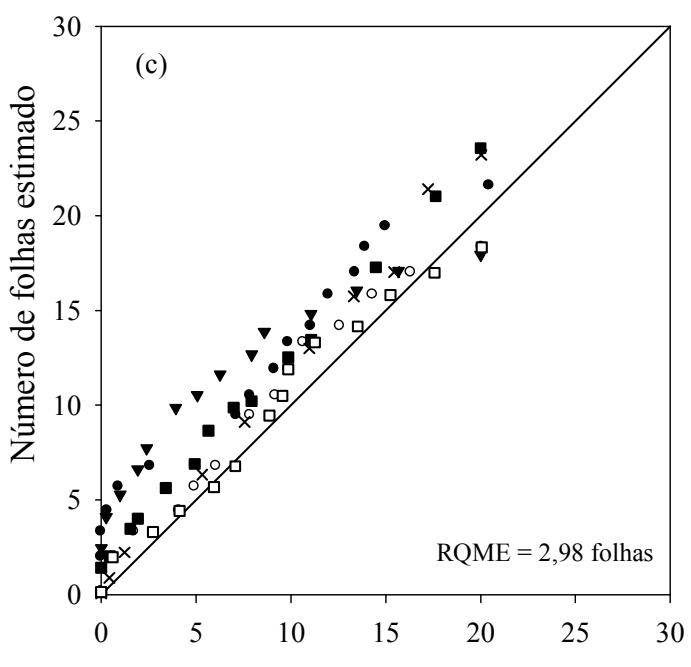

Número de folhas observado

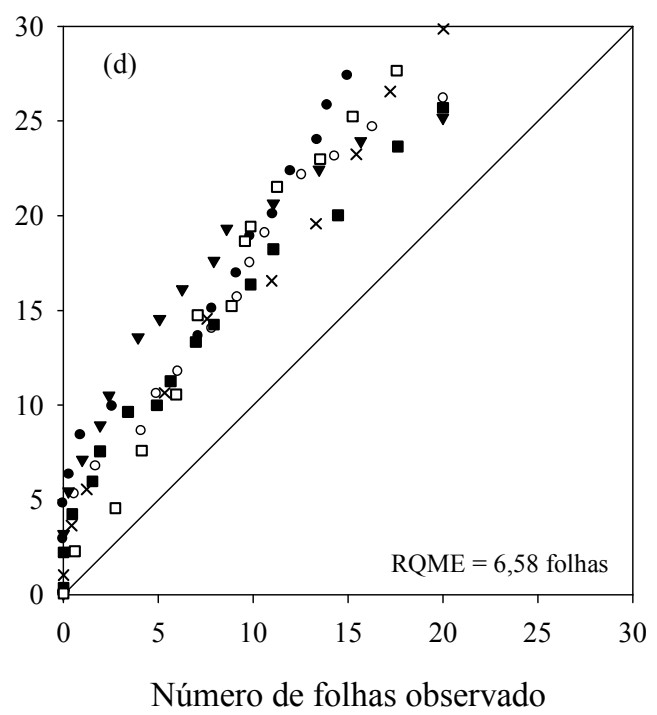

Figura 2 - Número de folhas estimado versus observado pelos modelos do Filocrono (a, c) e Wang e Engel (WE) (b, d), Maria da Fé (MG) 2010/2011. Os painéis a, b referem-se a cultivar 'Arbequina' e os painéis c,d referem-se a 'MGS ASC315'.

esse erro não causa nenhum impacto considerável na predição de outros processos baseados no número de folhas, inclusive na determinação da época de plantio da muda no campo.

Dessa forma, deve ser dada preferência ao modelo de WE para a 'Arbequina' e ao modelo do Filocrono para a 'MGS ASC315'. O modelo de WE também foi melhor na estimativa do aparecimento de folhas em espécies perenes como o eucalipto (Martins e Streck, 2007) e em espécies anuais como trigo (Xue et al., 2004), melão (Streck et al., 2006), batata (Streck et al., 2007b) e milho (Streck et al., 2008a, Streck et al., 2009).

O modelo de WE possui a vantagem de grande aplicabilidade em modelos de desenvolvimento, pois pode ser aplicado em condições de grande variação de temperatura do ar (Xue et al., 2004). Apesar do grande número de coeficientes, todos têm significado biológico (Wang e Engel, 1998;
Martins e Streck, 2007), sendo que a resposta biológica do desenvolvimento costuma ser não linear (Streck et al., 2003).

Mesmo com as vantagens do modelo de WE, o modelo do Filocrono foi superior para a cultivar de oliveira 'MGS ASC315', mesmo resultado encontrado para outras espécies anuais como o arroz (Streck et al., 2008b). Além disso, o modelo do Filocrono continua sendo utilizado para caracterizar o desenvolvimento vegetativo de plantas como batata (Dellai et al., 2005), arroz (Streck et al., 2007a), trigo (Rosa et al., 2009), entre outras. As principais vantagens do Filocrono se devem ao pequeno número de coeficientes (Xue et al., 2004), sua facilidade de aplicação, porém, a principal desvantagem é a não linearidade do desenvolvimento próximo às temperaturas cardinais, principalmente em torno da temperatura ótima (Martins e Streck, 2007). 


\section{CONCLUSÕES}

O modelo de Wang e Engel proporciona estimativa coerente do desenvolvimento foliar para a cultivar de oliveira 'Arbequina' e o modelo do Filocrono para a cultivar 'MGS ASC 315 '.

\section{AGRADECIMENTOS}

Ao Conselho Nacional de Desenvolvimento Científico e Tecnológico $(\mathrm{CNPq})$ e em parte à Fundação de Amparo à Pesquisa do Estado de Minas Gerais (FAPEMIG), referente ao projeto APQ-01392-13, pelo apoio financeiro e auxílio concedido ao primeiro e segundo autor. À Empresa de Pesquisa Agropecuária de Minas Gerais (EPAMIG) por toda a infraestrutura disponibilizada.

\section{REFERÊNCIAS BIBLIOGRÁFICAS}

ACEBEDO, M. M.; LAVEE, J.; LIÑÁN, A.; TRONCOSO, A. In vitro germination of embryos for speeding up seedling development in olive breeding programmes. Scientia Horticulturae, v.69, n.3-4, p.207-215, 1997.

AYERZA, R.; SIBBETT, G.S. Thermal adaptability of olive (Olea europaea L.) to the Arid Chaco of Argentina. Agricultural and Forest Meteorology, v.84, n.3, p.277285, 2001.

CAMARGO, A.P. de; SENTELHAS, P.C. Avaliação do desempenho de diferentes métodos de estimativa da evapotranspiração potencial no estado de São Paulo, Brasil. Revista Brasileira de Agrometeorologia, v.5, n.1, p.89-97, 1997.

DELLAI, J.; TRETIN, G.; BISOGNIN, D. A.; STRECK, N. A. Filocrono em diferentes densidades de plantas de mandioca. Ciência Rural, v.35, n.6, p.1269-1274, 2005.

DENNEY, J.O. McEACHERN, G.R.; GRIFFITHS, J.F. Modeling the termal adaptability of the olive (Olea europaea L.) in Texas. Agricultural and Forest Meteorology, v.35, n.1-4, p.309-327, 1985.

EMPRESA BRASILEIRA DE PESQUISAAGROPECUÁRIA. Cultivo de Oliveira. Sistemas de produção 16: EMBRAPA Clima Temperado. Pelotas, 2009. Disponível em: <http:// www.cpact.embrapa.br>. Acesso em: 12 jun. 2013.

EVERS, J.B.; VOS, J.; FOURNIER, C.; ANDRIEU, B.; CHELLE, M.; STRUIK, P.C. An architectural model of spring wheat: evaluation of the effects of population density and shading on model parameterization and performance. Ecological Modelling, v.200, n.3-4, p.308-320, 2007.

GOULAS, V.; EXARCHOU, V.; TROGANIS, A. N.; PSOMIADOU, E.; FOTSIS, T.; BRIASOULIS, E.;
GEROTHANASSIS, I. P. Phytochemicals in olive-leaf extracts and their antiproliferative activity against câncer and endotelial cells. Mollecular Nutricional \& Food Research, v.53, n.5, p.600-608, 2009.

INTERNATIONAL OLIVE COUNCIL. 2012. Online. Disponível em: < http://www.internationaloliveoil.org/ estaticos/view/135-imports-by-selected-markets $>$ Acesso em: 7 jan. 2013.

KITSAKI, C.K.; ANDREADIS, E.; BOURANIS, D.L. Developmental events in differentiating floral buds of four olive (Olea europaea $\mathrm{L}$.) cultivars during late winter to early spring. Flora, v.205, n.9, p.599-607, 2010.

LEITE, H.G.; ANDRADE, V.C.L. de. Um método para condução de inventários florestais sem o uso de equações volumétricas. Revista Árvore, v.26, n.3, p.321-328, 2002.

LISBOA, P.M.M.; MARTINS, F.B.; ALVARENGA, M.I.N.; VIEIRA NETO, J.; REIS, D.F. da. Desenvolvimento vegetativo de duas cultivares de oliveira na fase de muda. Ciência Rural, v.42, n.9, p.1556-1562, 2012.

MARTINS, F.B.; REIS, D.F.; PINHEIRO, M.M.V. Temperatura base e Filocrono em duas cultivares de oliveira. Ciência Rural, v.42, n.11, p.1975-1981, 2012.

MARTINS, F.B.; SILVA, J.C.; STRECK, N.A. Estimativa da temperatura base para emissão de folhas e do Filocrono em duas espécies de eucalipto na fase de muda. Revista Árvore, v.31, n.3, p.373-381, 2007.

MARTINS, F. B.; STRECK, N. A. Aparecimento de folhas em mudas de eucalipto estimado por dois modelos. Pesquisa Agropecuária Brasileira, v.42, n.8, p.1091-1100, 2007.

ROSA, H. T. WALTER, L. C.; STRECK, N. A.; ALBERTO, C. M. Métodos de soma térmica e datas de semeadura na determinação de Filocrono de cultivares de trigo. Pesquisa Agropecuária Brasileira, v.44, n.11, p.13741382, 2009.

SOLTANI, A.; SINCLAIR, T.R. Modeling Physiology of crop development, growth and yield. Oxfordshire: $\mathrm{CAB}$ Internacional, 2012. $322 \mathrm{p}$.

STATSOFT, INC. Statistica for windows (data analysis softwares system) version 8.0. 2008.

STRECK, N.A. A generalized nonlinear air temperature response function for node appearance rate in muskmelon (Cucumis melo L.). Revista Brasileira de Agrometeorologia, v.10, n.1, p.105-111, 2002.

STRECK, N. A.; BELLÉ, R.A.; ROCHA, E.K.da; SCHUH, M. Estimating leaf appearance and phyllochron in safflower (Carthamus tinctorius L.). Ciência Rural, v.35, n.6, p.14481450, 2005.

STRECK, N.A. BOSCO, L.C.; LAGO, I. Simulating leaf appearance in Rice. Agronomy Journal, v.100, n.3., p.490-501, 2008b. 
STRECK, N.A.; GABRIEL, L.F.; SAMBORANHA, F.K.;LAGO, I.; SCHWANTES, A.P.; SCHONS, A. Comparing two versions of a non-linear model for simulating leaf number and developmental stages in maize based on air temperature. Ciência Rural, v.39, n.3, p.642-648, 2009.

STRECK, N.A.; GABRIEL, L.F.; BUSKE, T.C.; LAGO, I.; SAMBORANHA, F.K.; SCHWANTES, A.P. Simulating leaf appearance in a maize variety. Bioscience Journal, v.26, n.3, p. 384-393, 2010.

STRECK, N. A.; LAGO, I.; BURIOL, G. A.; HELDWEIN, A. B.; TIBOLA, T.A non-linear model to simulate node appearance in muskmelon (Cucumis melo L.) grown inside plastic greenhouse as a function of air temperature. Revista Brasileira de Agrometeorologia, v.14, n.2, p.210-216, 2006.

STRECK, N.A.; LAGO, I.; GABRIEL, L.F.; SAMBORANHA, F.K. Simulating maize phenology as a function of air temperature with linear and nonlinear model. Pesquisa Agropecuária Brasileira, v.43, n.4, p.449-455, 2008a.

STRECK, N.A.; LAGO, I.; PAULA, F.L.M.de; BISOGNIN, D.A.; HELDWEIN, A.B. Improving predictions of leaf appearance in field grown potato. Scientia Agrícola, v.64, n.1, p.12-18, $2007 \mathrm{~b}$.

STRECK, N.A.; MICHELON, S.; ROSA, H.T.; WALTER, L.C.; BOSCO,L.C.; PAULA, G.M. de; CAMERA, C.; SAMBORANHA, F.K.; MARCOLIN, E.; LOPES, S.J. Filocrono de genótipos de arroz irrigado em função da época de semeadura. Ciência Rural, v.37, p.323-329, 2007a.
STRECK, N.A.; WEISS, A.; XUE, Q.; BAENZIGER, P.S. Incorporating a chronology response function into the prediction of leaf appearance rate in winter wheat. Annals of Botany, v.92, p.181-190, 2003.

TERAMOTO, J. R. S.; BERTONCINI, E. I.; PRETAPANTANO, A. Mercado dos produtos da oliveira e os desafios brasileiros. Informações Econômicas, v.43, n.2, 2013.

VIEIRA NETO, J.; OLIVEIRA, A.D.de.; CAPRONI, C.M.; VILLA, F.; SILVA, L.F.O.da. Desempenho de jardins clonais de oliveira (Olea europaea) em cortes sucessivos visando a sua propagação por estaquia. Cerne, v.17, n.1, p.117-122, 2011.

WANG, E.; ENGEL, T. Simulation of phonological development of wheat crops. Agricultural Systems, v.58, n.1, p.1-24, 1998.

WILlmotT, C.J. On the validation models. Physical Geography, v.2, n.1, p.184-194, 1981.

XUE, Q.; WEISS, A.; BAENZIGER, P.S. Predicting leaf appearance in field-grown winter wheat: evaluating linear and non-linear models. . Ecological Modelling, v.175, p.261-270, 2004.

YAN, Q.; HUNT, L.A. An equation for modelling the temperature response of plants using only the cardinal temperatures. Annals of Botany, v.84, n.5, p.607-614, 1999. 